\title{
Endophenotype strategies for the study of neuropsychiatric disorders: A quest
}

The identification of genes that contribute to the susceptibilities to complex neuropsychiatric disorders such as schizophrenia, major depression and bipolar disorders has not been as successful using conventional genetic approaches as had been hoped. There are several problems associated with the conventional approaches, including the validity of psychiatric diagnosis itself, excluding carriers of relevant genes who cannot be identified in the absence of manifest symptoms, and the heterogeneity of neuropsychiatric disorders, both clinically and etiologically. A new direction that appears encouraging is the identification of neurobiological and neurobehavioural characteristics, hidden from the "naked eye" associated with these complex neuropsychiatric disorders. These characteristics are termed endophenotypes, that may be more closely linked to gene expression in the causal chain from genes to psychiatric diagnosis disorders [1,2]. An endophenotype is a special kind of biomarker, one that is not the consequence of illness and is heritable. It plays an important role for bridging the gap between the microscopic level (e.g., molecular genetics) and the macroscopic level (e.g., clinical symptoms) of neuropsychiatric disorders such as schizophrenia and bipolar disorders $[3,4]$. The identification of endophenotypes is very crucial to the identification of genes that predispose someone to neuropsychiatric disorders. Therefore, the study of endophenotype is of particular usefulness for us to understand the underlying mechanism of the illness process of neuropsychiatric disorders, aiding the clinicians to make accurate diagnosis and for early detection purposes. The Director of the United States National Institute of Mental Health (NIMH), Thomas R. Insel and his associate Dr. Bruce N. Cuthbert have provided an ideal overview of the importance of endophenotypes in the context of the mission of the NIMH [5] and have provided pointers [6] to exciting developments in revising diagnostic practices that utilize endophenotypes.

A substantial number of studies, especially of at-risk off-spring of patients, have suggested that neurocognitive dysfunctions are among the most promising of the candidate endophenotypes. With the completion of the human genome project, the main hurdle for scientists working with neuropsychiatric disorders for the next decade will be how to characterize the manifold human endophenotypes from the molecular level to the mind level, and most importantly, detecting unharmonious heritable contributors to the mind in neuropsychiatric disorders. The development of cognitive neurosciences, neuropsychology and imaging genetics - a strategy for mapping neural structures and brain activity as a function of genotype in living humans - has encouraged a conceptual transformation by showing that the greater power of endophenotypes lies in using genetic risk variants as tools for discovery of the mediating neural mechanisms that bridge the gap from DNA sequence to pathological behaviour.

In October 11th-13th 2010, with support from the National Basic Research Program of China (973 Program) (2007CB512302), The Key Laboratory of Mental Health of the Institute of Psychology, Chinese Academy of Sciences has organized a strategic symposium for learning about endophenotypes, titled "Endophenotype strategy for psychotic disorders and summit meeting of Key Laboratory of Mental Health, Institute of Psychology, CAS". It was a 3-d symposium taking place in Beijing. Participation was by invitation only, and a group of internationally renowned scholars and clinicians were

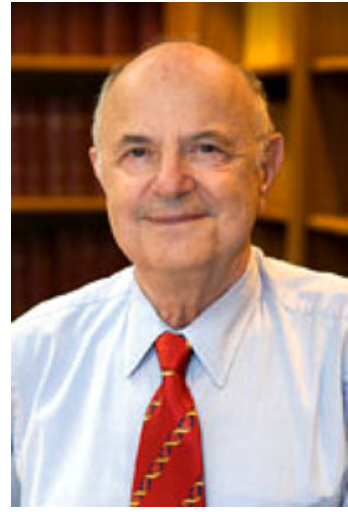

Professor Irving I. Gottesman

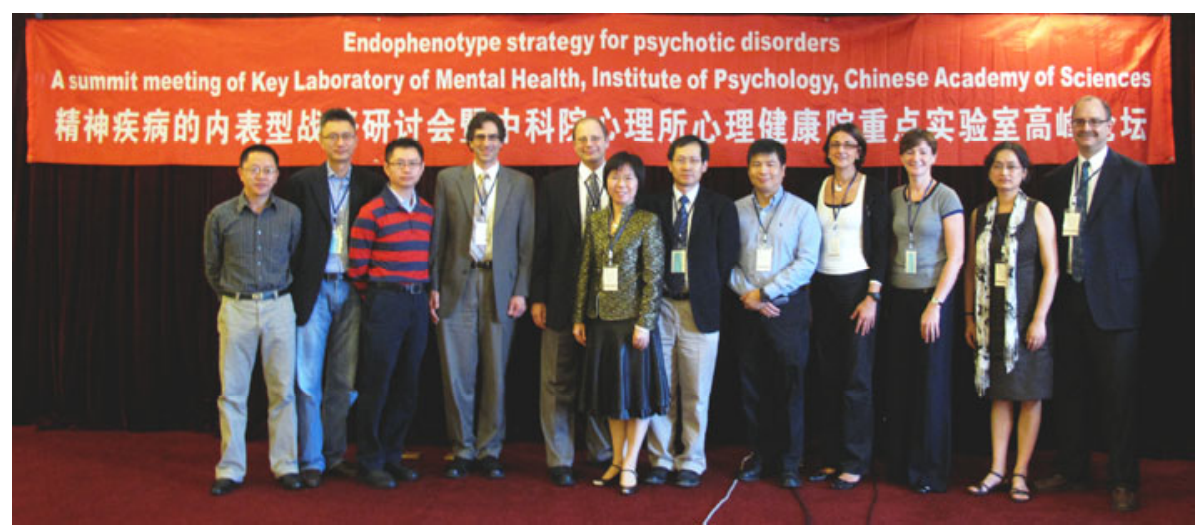

A summit meeting of Key Laboratory of Mental Health, Institute of Psychology, Chinese Academy of Sciences 
invited, including Prof. David Glahn from Yale Medical School, Prof. Bill Honer from University of British Columbia, Prof. Pak Sham from the University of Hong Kong, Prof. Bill Stone from Harvard Medical School, Dr. Paola Dazzan from the Institute of Psychiatry (London), Dr. Grainne McAlonan from the University of Hong Kong, Dr. Xaolu Hsi from Boston, and Dr. Eric Cheung from Hong Kong as well as our local scholars and peer colleagues. Professor Irving Gottesman, the founder of the endophenotype concept and strategy for neuropsychiatric disorders, participated by giving a video-taped lecture to all the participants.

The main purpose of this special topic is to document the work we have presented or will be doing in the area of endophenotypes for neuropsychiatric disorders, and to make the information available to a worldwide audience of neuroscientists and clinicians. In particular, this special issue comprises 9 papers-which were presented at the Symposium. The first 4 papers [7-10] are mainly focus on neuroanatomical and neuroimaging issues of endophenotypes for schizophrenia and related disorders. Two papers $[11,12]$ are working on the development of a battery specifically capturing neuropsychological endophenotypes for schizophrenia, and how it may help develop a research-oriented programme for studying endophenotypes for neuropsychiatric disorders. There are two papers $[13,14]$ specifically dealing with the analysis issues including novel pathway-based analysis for genome-wide association studies for schizophrenia, and the issues we should pay attention to when applying statistics for endophenotype study. Finally, there is one paper [15] working on the establishment of a consortium for endophenotypes to study neuropsychiatric disorders in mainland China. In particular, this paper illustrates a potential example using neurological soft signs in following the steps for building such a consortium.

In sum, the use of endophenotypes is a powerful tool to index intermediate neurobiological processes that are influenced by genetic variation. It allows us to go through the most exciting period of studying complex neuropsychiatric disorders in the coming decade and to shed light on to the potential roles for contributing to this promising research field.

1 Gottesman I I, Shields J. Genetic theorizing and schizophrenia. Br J Psychiatry, 1973, 122: 15-30

2 Gottesman I I, Shields J. Schizophrenia and Genetics: A Twin Study Vantage Point. New York and London: Academic Press, 1972

3 Gottesman I I, Gould T D. The endophenotype concept in psychiatry: Etymology and strategic intentions. Am J Psychiatry, 2003, 160: 636-645

4 Chan R C K, Gottesman I I, Ge X, et al. Strategies for the study of neuropsychiatric disorders using endophenotypes in developing countries: A potential databank from China. Front Hum Neurosci, 2010, 4: 207

5 Insel T R, Cuthbert B N. Endophenotypes: Bridging genomic complexity and disorder heterogeneity. Biol Psychiatry, 2009, 66: 988-989

6 National Institute of Mental Health. The National Institute of Mental Health Strategic Plan. Bethesda, MD: National Institute of Mental Health. NIH publication 08-6368. Available at: http://www.nimh.nih.gov/about/strategic-planning-reports/index.shtml. Accessed October 17, 2009

7 Honer W G, Lang D J, Kopala L C, et al. First episode psychosis with extrapyramidal signs prior to antipsychotic drug treatment. Chinese Sci Bull, 2011, 56: 3361-3371

8 Dazzan P, Chan R C K. Which neuroloigcal abnormalities and neuropsychological impairments share the same substrate in psychosis? Chinese Sci Bull, 2011, 56: 3372-3375

9 McAlonan G M, Yu K K, Chan R C K, et al. Is there an anatomical endophenotype for neurodevelopmental disorders? A review of dual disorder anatomical likelihood estimation (ALE) meta-analyses of grey matter volumes. Chinese Sci Bull, 2011, 56: 3376-3381

10 Glahn D C, Blangero J. Why endophenotyp development requires families? Chinese Sci Bull, 2011, 56: 3382-3384

11 Stone W S, Hsi X. Recent developments in neuropsychological endophenotypes for schizophrenia: Development of the MATRICS battery, liability syndromes and the near future. Chinese Sci Bull, 2011, 56: 3385-3393

12 Lui S S Y, Sham P, Chan R C K, et al. A family study of endophenotypes for psychosis within an early intervention programme in Hong Kong: Rationale and preiminary findings. Chinese Sci Bull, 2011, 56: 3394-3397

13 Zhang K, Zhang L Y, Zhang W N, et al. Pathway-based analysis for genome-wide association studies of schizophrenia to provide new insight in schizophrenia study. Chinese Sci Bull, 2011, 56: 3398-3402

14 Sham P C, Cherny S, Hall M H. Statistical issues and approaches in endophenotype research. Chinese Sci Bull, 2011, 56: 3403-3408

15 Chan R C K. Consortium for the Human Information and Neurocognitive Endophenotype (CHINE) in mainland China: An example from neurological soft signs for neuropsychiatric disorders. Chinese Sci Bull, 2011, 56: 3409-3415

Special Topic Editors CHAN Raymond Chor-kiu Neuropsychology and Applied Cognitive Neuroscience Laboratory, Key Laboratory of Mental Health, Institute of Psychology, Chinese Academy of Sciences, Beijing 100101, China

GOTTESMAN Irving. I

Departments of Psychology and Psychiatry, University of Minnesota, Minneapolis, United States of America

FU Xiao-Lan

Institute of Psychology, Chinese Academy of Sciences, Beijing 100101, China

Open Access This article is distributed under the terms of the Creative Commons Attribution License which permits any use, distribution, and reproduction in any medium, provided the original author(s) and source are credited. 